\title{
Optimised COST-231 Hata Models for WiMAX Path Loss Prediction in Suburban and Open Urban Environments
}

\author{
Mardeni.R \\ Faculty of Engineering, Multimedia University \\ Jalan Multimedia, 63100 Cyberjaya, Malaysia \\ Tel: 60-3-8312-5481Ｅ-mail: mardeni.roslee@mmu.edu.my \\ T. Siva Priya (Corresponding author) \\ Faculty of Engineering, Multimedia University \\ Jalan Multimedia, 63100 Cyberjaya, Malaysia \\ Tel: 60-12-287-6023 E-mail: sivapriya.thiagarajah@gmail.com
}

\begin{abstract}
In Malaysia, the incumbent WiMAX operator utilises the bands of $2360-2390 \mathrm{MHz}$ to provide broadband services. Like all Radio Frequency (RF), WiMAX is susceptible to path loss. In this paper, field strength data collected in Cyberjaya, Malaysia is used to calculate the path loss suffered by the WiMAX signals. The measured path loss is compared with the theoretical path loss values estimated by the COST-231 Hata model, the Stanford University Interim (SUI) model and the Egli model. The best model to estimate the path loss based on the path loss exponents was determined to be the COST-231 Hata model. From this observation, an optimised model based on COST-231 Hata parameters is developed to predict path loss for suburban and open urban environments in the $2360-2390 \mathrm{MHz}$ band. The optimised model is validated using standard deviation error analysis, and the results indicate that the new optimised model predicts path loss in both suburban and open urban environments with very low standard deviation errors of less than $4.3 \mathrm{~dB}$ and $1.9 \mathrm{~dB}$ respectively. These values show that the model optimisation was done successfully and that the new optimised models will be able to determine the path loss suffered by the WiMAX signals more accurately. The optimised model may be used by telecommunication providers to improve their service.
\end{abstract}

Keywords: Model Optimisation, Path Loss Models, Path Loss Exponents, WiMAX

\section{Introduction}

In Malaysia, incumbent WiMAX operator Packet One Networks (P1) Sdn. Bhd. utilises the bands of $2360-2390 \mathrm{MHz}$ to provide broadband services. Fixed WiMAX services are beneficial to the development of broadband used by consumers and small businesses while mobile WiMAX may be used for mobile services being provisioned by existing fixed-line carriers that do not own a $3 \mathrm{G}$ spectrum to provide Voice-over-IP (VoIP) or mobile entertainment services [Senza Filli Consulting, 2005].

Non-Line of Sight (NLOS) between a transmitter and a receiver in a wireless link will introduce multipath, which decreases the signal strength and introduces a subsequent increase in the receiver Bit Error Rate (BER) [Rappaport, T.S., 2002]. The path loss may differ in severity depending on the terrain and whether it is a rural, suburban or urban environment [Rappaport, T.S., 2002]. To increase the robustness of the transmitted information, engineers need to estimate the path loss introduced by a terrain over which the signal will propagate to sufficiently compensate for the power lost during signal propagation. Existing path loss models may be used to estimate this path loss, but it is ideal to develop an optimised model to use over a certain terrain in a particular band for faster transmitter power estimation.

In a previous study, Abhayawardhana et al. (2005) had conducted a feasibility study on the use of empirical models to predict path loss in BWA in the $3.5 \mathrm{GHz}$ band. Similar studies were also conducted by Rial et al. (2007) and Belloul et al. (2009).

Cyberjaya, in the district of Sepang, Selangor, has a mostly suburban terrain profile. In the recent years, Cyberjaya's natural suburban terrain profile has seen a rapid increase in the construction of three to four storey buildings that cater for the booming multinational companies here [Town \& Country Planning Department, Malaysia, 2000]. These buildings, along with a multitude of wide roads, car parks and pedestrian pavements, have given rise to an environment which is slightly more than suburban. For this study, the terrain profile where the field testing was conducted was categorized carefully to be either "open urban" (multiple-story buildings 
situated rather close to roads and car parks) or suburban (small hillocks with medium height trees) .The "open urban" profile is not considered to be the typical urban environment where skyscrapers and high rise buildings are packed close to each other.

In this paper, two optimised models to predict path loss for WiMAX signals in the $2360-2390 \mathrm{MHz}$ based on the COST-231 Hata model [COST Action 231, 1999] will be introduced. The optimised path loss models will be developed based on comparison between the measured path loss and the path loss estimated by the COST-231 Hata model. The COST-231 Hata model is selected because it showed the best agreement with the measured path loss in terms of path loss exponent, as compared to the Stanford University Interim SUI model introduced by Erceg,V. \& Hari, K.V. S. (2001) and the Egli path loss model introduced by Egli (1957). The performances of these optimised models in estimating the path loss in the $2360-2390 \mathrm{MHz}$ band in both suburban and open urban environments are validated using standard deviation errors analysis.

\section{Data collection and field setup}

The base station (BS) in the Multimedia University, herein to be known as MMU BS, is situated 23m above ground level and has four WiMAX Base Stations (WBS) and serves a mix of open urban and suburban environments. Each WBS has a transceiver sectorized antenna which transmits in vertical polarization. The Customer Premise Equipment (CPE) used is a vertically polarized directional antenna with a $50^{\circ}$ beamwidth. It was mounted on a makeshift mast, and was adjustable to $2 \mathrm{~m}$ and $4 \mathrm{~m}$ heights (Figure 1). The field strength was observed using a spectrum analyzer at CPE heights of $2 \mathrm{~m}$ and $4 \mathrm{~m}$ for the duration of 1 minute and was recorded at every 10 s interval.

The field testing was conducted within a $1 \mathrm{~km}$ radius from the site, which is the estimated coverage of the MMU BS (Figure 2). The field strength was collected along the Line of Sight (LOS) of each WBS. At each measurement location, a Global Positioning System (GPS) was used to establish the location of the CPE and a compass was used to confirm that the antenna was facing towards the LOS path of the selected WBS. The measurements were taken at every $50 \mathrm{~m}$ radial increment within the coverage hexagon (Figure 2). Due to geographical limitations caused by dense jungle, the maximum radial increment measurements recordable at the $270^{\circ} \mathrm{WBS}$ are $500 \mathrm{~m}$.

At each measurement location, the terrain profile was observed and categorised to be either suburban or open urban. All the field data from the four WBSs was then collectively separated to either suburban or open urban data and tabulated for the ensuing analysis.

\section{Path loss models}

\subsection{Egli model}

The Egli model is suitable for use in mobile systems in the bands of $3 \mathrm{MHz}-3 \mathrm{GHz}$ and is normally used when there is LOS between one fixed antenna and one mobile antenna [Egli, 1957]. This model is selected for this study as the Egli model can be used for path loss prediction in the frequency range selected for this study.

The Egli path loss is calculated using (1) [Egli, 1957]

$$
P L(d B)=G_{B} G_{M}\left[\frac{h_{B}}{d^{2}} h_{M}\right]^{2}\left[\frac{40}{f}\right]^{2}
$$

where $G_{B}$ is the gain of the BS antenna, $G_{M}$ is the gain of the CPE, $h_{B}$ is the height of the BS antenna from ground level, $h_{M}$ is the height of the CPE, $d$ is the receiver distance from the BS and $f$ is the operating frequency of the CPE in MHz. The Egli model can be used when there is propagation over irregular terrain [Egli, 1957]. It should be noted that the Egli model does not provide correction factors for different environments.

\subsection{COST-231 Hata model}

The COST-231 Hata model is an extension of the Hata-Okumura model developed by Hata(1981) from the original Okumura path loss model [Okumura, 1968] and is used for the prediction of path loss for mobile wireless systems in urban environments. Correction factors for the use of this model in suburban environments are provided in [Abhayawardhana et al., 2005]. This model was developed for use in 1500-2000MHz with CPE heights up to $10 \mathrm{~m}$ and transmitter heights of 30-200m [Hata, 1981]. However, due to its simplicity and extensive usage, this model is selected for this study in the $2360-2390 \mathrm{MHz}$ band. Furthermore, this model is the basis for the Standard Propagation Model which is used for path loss modelling in WiMAX systems [Asztalos, 2008].

The COST-231 Hata model path loss is calculated using (2) [COST Action 231, 1999]

$$
P L(d B)=46.3+33.9 \log _{10}(f)-13.82 \log _{10}\left(h_{b}\right)-a h_{m}+\left(44.9-6.55 \log _{10}\left(h_{b}\right)\right) \log _{10} d+c_{m}
$$


where $f$ is the frequency in MHz, $d$ is the distance between BS and CPE antennae in km and $h_{b}$ is the BS antenna height above ground level in meters.

The correction parameter $a h_{m}$ is defined by (3) and (4) for urban and suburban environments respectively [Abhayawardhana et al., 2005]. The correction parameter, $c_{m}$ is given as $c_{m(u r b a n)}=3 \mathrm{~dB}$ and $c_{m(\text { suburban })}=0 \mathrm{~dB}$ [Abhayawardhana et al., 2005].

$$
\begin{aligned}
& a h_{m}=3.2\left(\log _{10}\left(11.75 H_{r}\right)\right)^{2}-4.97 \\
& a h_{m}=\left(1.1 \log _{10} f-0.7\right) H_{r}-\left(1.56 \log _{10} f-0.8\right)
\end{aligned}
$$

where $H_{r}$ is the height of the CPE antenna in meters.

\subsection{Stanford University Interim (SUI) model}

The SUI model was developed under the Institute of Electrical and Electronics Engineers (IEEE) 802.16 working group for prediction of path loss in urban, suburban and rural environments [Erceg,V. \& Hari, K. V. S., 2001]. The applicability of this model in the $2.3 \mathrm{GHz}$ band has not been validated. However, due to the availability of correction factors for the operating frequency, this model is selected for this study.

The SUI model path loss is calculated using (5) [Erceg, V. \& Greenstein, L. J., 1999]

$$
P L(d B)=A+10 \gamma \log _{10}\left(\frac{d}{d_{o}}\right)+X_{f}+X_{h}+s
$$

where $d_{o}=100 \mathrm{~m}, d$ is the distance between BS and CPE antenna in meters, and $s$ is a log-normally distributed factor used to account for tree and clutter shadowing. The values given for $s$ in [9] are between $8.2 \mathrm{~dB}$ and 10.6dB.

The path loss exponent for the SUI model, $\gamma$, is determined from constants (Table 1), which were developed through studies done by Erceg, V. \& Greenstein, L. J. (1999). In this model, three types of terrains are used. This paper does not show the constants for Terrain $\mathrm{C}$ which depicts rural conditions. Terrain $\mathrm{A}$ is used for maximum path loss, depicting urban conditions, while terrain B is used for hilly terrains with light tree densities, depicting suburban conditions.

The path loss exponent, $\gamma$ is determined by (6) [Erceg, V. \& Greenstein, L. J., 1999]

$$
\gamma=a-b h_{b}+\frac{c}{h_{b}}
$$

where $h_{b}$ is the height of the BS in meters and should be between $10 \mathrm{~m}$ and $80 \mathrm{~m}$ above ground level.

Parameter $A$ is known as the intercept parameter [Erceg, V. \& Greenstein, L. J., 1999] and is defined as

$$
A=20 \log _{10}\left(\frac{4 \pi d_{0}}{\lambda}\right)
$$

The SUI model also provides correction factors for the operating frequency, $X_{f}$, and the CPE antenna height, $X_{H}$, that can be found using (8) and (9) [Erceg,V. \& Hari, K. V. S., 2001],

$$
\begin{gathered}
X_{f}=6.0 \log _{10}\left(\frac{f}{2000}\right) \\
X_{H}=-10.8 \log _{10} \frac{H_{r}}{2000} \text { for Terrains } A \text { and } B
\end{gathered}
$$

where $H_{r}$ is the CPE antenna height in meters and $f$ is the operating frequency in $\mathrm{MHz}$.

\section{Measured path loss determination}

The rate of propagation path loss with respect to a distance is shown by the path loss exponent. If the path loss exponent value is 2 , then the environment propagation characteristic is close to free space propagation [Abhayawardhana et al., 2005], or one that has less clutter. A path loss of 2- 4 indicates an environment that is urban [Rao et al., 2000].

The path loss exponent is determined using (10) [Wikipedia, 2010],

$$
P L(d B)=10 n \log _{10} d
$$

where $d$ is the distance from the transmitter and $n$ is the path loss exponent. The equation given in (10) can be manipulated to determine the value of the path loss exponent of an environment. From (10), when a graph of path loss is plotted against the distance $(\mathrm{dB})$, then the path loss exponent, $n$, can be determined by calculating the slope of this graph.

The path loss at a given location with respect to the path loss at a reference distance, $d_{o}$, may be determined using the Least Square (LS) regression analysis shown in (11) [Abhayawardhana et al., 2005], 


$$
P L_{d}(d B)=P L_{d_{o}}+10 n \log _{10}\left(\frac{d}{d_{o}}\right)
$$

where $d_{o}$ is the reference point at $100 \mathrm{~m}$ and $n$ is the path loss exponent. The path loss exponent obtained from the slope of the Path Loss (dB) versus $\log d$ graph using (10) is substituted into (11) to determine the actual (measured) path loss of the area within $1 \mathrm{~km}$ from the MMU BS.

The measured path loss $(\mathrm{dB})$ was then plotted against the distance (Figure 3) and the slope was calculated to determine the path loss exponents [Wikipedia, 2010] in open urban and suburban environments for CPE heights of $2 \mathrm{~m}$ and $4 \mathrm{~m}$.

The measured Received Signal Strength (RSS), as well as the calculated path loss using (1), (2), (5) and (11) is presented at the end of this paper (Table 2).

When the path loss is plotted against distance (Figure3), it was shown that path loss increased as the distance between the transmitter and the receiver is increased and that the path loss at a given distance is more in open urban environments than in suburban environments. Higher path loss is expected to be experienced by a CPE which is further away from the BS because the signal experiences more multipath fading as it propagates further away from the transmitter [Rappaport, 2002]. Higher path loss is also expected in open urban conditions because the clutter of the buildings will cause multipath fading and signal strength deterioration, in comparison to suburban conditions in which a LOS between transmitter and receiver may exist and allow the signal to propagate without suffering from diffractions, reflections, absorption and scattering [Anderson, 2003]. The path loss exponents found from the graphs (Figure 3) are summarized (Table 3) and compared against the theoretical path loss exponents which are calculated from the path loss models.

\section{Best model selection}

The path loss estimated by the SUI, COST-231 Hata and Egli models are calculated, and plotted against distance on the same graph as that of the measured path loss (Figures 4 and 5).

The path loss exponents found from the slopes of these graphs (Figures 4 and 5) are subsequently summarised (Table 4). The summary (Table 4) shows that the SUI and Egli models over-predict the path loss exponents of fixed WiMAX receivers in the suburban and open urban environments in the $2360-2390 \mathrm{MHz}$ band. For both open urban and suburban environments, the path loss exponent estimated by the COST-231 Hata model is in closest agreement with the LS analysis, which shows the actual path loss characteristics in Cyberjaya. This is especially true for the open urban environment. Based on this, the COST-231 Hata model is selected as the best model for optimisation.

Rao et al., (2000) states that the standard deviation of error between the measured path loss and the path loss predicted can be estimated by (12)

$$
P L_{\text {error }}=\left|P L_{\text {measured }}-P L_{\text {model }}\right|
$$

where $P L_{\text {error }}$ is the standard deviation of measured path loss, $P L_{\text {measured }}$, with the estimated path loss of models, $P L_{\text {model }}$.The standard deviation of error analysis will be used to analyse the performance of the new path loss models optimised from the existing COST-231 Hata path loss model.

\section{COST-231 Hata model optimisation}

Based on closest agreement with measured path loss exponents (Table 4), the COST-231 Hata model is selected as a basis for optimisation to develop a new model for the prediction of path loss for fixed WiMAX receivers in the $2360-2390 \mathrm{MHz}$ band. This section of the paper describes how the COST-231 Hata model is first optimised to match the measured path loss and then a comparison analysis of the performance of the new optimised model is madr against the measured path loss and the path loss estimated by the COST-231 Hata model.

\subsection{Optimisation of measured data into COST-231 Hata model}

The optimised model is developed based on the frequency of $2375 \mathrm{MHz}$, which is the center frequency for the band of $2360-2390 \mathrm{MHz}$. The height of the BS is taken as $23.6095 \mathrm{~m}$, which is the height of the building plus the height of half of the sectorised antenna length.

The COST-231 Hata model equation as shown by (2) of this paper which consists of three basic elements like in any other empirical propagation models, as described by Jacques, L. \& Michel, S., (2000). The entire equation can be grouped into the initial offset parameter, $E_{o}$, the initial system design parameter, $E_{s y s}$, and the slope of the model curve, $\beta_{\text {sys }}$.

The COST-231 Hata model from (2) can be expressed by (13), (14) and (15). 


$$
\begin{aligned}
E_{o} & =46.3-a h_{m}+c_{m} \\
E_{\text {sys }} & =33.9 \log _{10}(f)-13.82 \log _{10}\left(h_{b}\right) \\
\beta_{\text {sys }} & =\left(44.9-6.55 \log _{10}\left(h_{b}\right)\right) \log _{10} d
\end{aligned}
$$

where total path loss is given by Jacques, L. \& Michel, S.,(2000) as

$$
P L(d B)=E_{o}+E_{\text {sys }}+\beta_{\text {sys }}
$$

The path loss calculated from the measured data and the COST-231 Hata model are plotted and a simple logarithmic curve is used to plot the differences between the measured path loss and the path loss estimated by the COST-231 Hata model (Figures 6 and 7). The new logarithmic curve was subsequently presented as the optimised models. The new logarithmic curves, entitled "optimised model" (Figures 6 and 7) are in the form of

$$
y=a \ln (x)+b
$$

Based on (17), $y$ is taken as the path loss (PL) and $\ln (x)$ depicts the relationship of distance from transmitter in meters. $a$ is taken to be the $\beta_{s y s}$ and $b$ is taken to be a cumulative of $E_{o}$ and $E_{s y s}$.

Based on the explanations subsequent to (17), two new equations (18) and (19) are presented as optimised models for the prediction of path loss in suburban and open urban environments respectively in the 2360-2390MHz.

An optimised model for predicting path loss for WiMAX based on COST-231 Hata model for suburban environment in $2360-2390 \mathrm{MHz}$ is presented as

$$
P L(d B)=36.2+9.467 \ln (x)
$$

An optimised model for predicting path loss for WiMAX based on COST-231 Hata model for open urban environment in $2360-2390 \mathrm{MHz}$ is presented as

\subsection{Optimised model performance analysis}

$$
P L(d B)=8.595+14.53 \ln (x)
$$

The path loss is calculated using (18) and (19) and plotted against the measured path loss and the COST-231 Hata predicted path loss (Figures 8 and 9). Accordingly, the new path loss exponent estimated from the slopes of these figures for the optimised model in the suburban and open urban environments are summarised (Table 5).

In the suburban environment, the path loss estimated by the optimised model follows the measured path loss closely (Figure 8). Based on the summary of path loss exponents (Table 5), the path loss exponent predicted by the optimised model is lower than the measured path loss exponent for CPE heights of $2 \mathrm{~m}$ and $4 \mathrm{~m}$. However, it can be concluded that the prediction of the optimised model (Table 5) increases in accuracy as the CPE height is increased.

In the open urban environment, the path loss estimated by the optimised model follows the measured path loss closely (Figure 9). Based on the summary (Table 5), the path loss exponent predicted by the optimised model is almost the same as the measured path loss exponent for CPE at $2 \mathrm{~m}$. For CPE at $4 \mathrm{~m}$, the predicted path loss exponent from the optimised model is higher than the measured path loss. This indicates that the accuracy of the optimised model's prediction in terms of path loss exponent reduces as the CPE height is increased.

A standard deviation error analysis is done and summarised (Table 6) to validate the performance of the optimised models. In the suburban environment, the standard deviation of error between measured and predicted path loss ranges from $0.3-4.3 \mathrm{~dB}$ and 1.1-4.2dB for CPE heights of $2 \mathrm{~m}$ and $4 \mathrm{~m}$ respectively. It can be seen that the accuracy of the prediction by the optimised models increases with the CPE distance from the transmitter. The model also predicts the path loss better at a higher CPE height, which is consistent with the discussions in terms of accuracy of path loss exponent prediction (Table 5).

In the open urban environment, the standard deviation of error between measured and predicted path loss ranges from $0.01-0.1 \mathrm{~dB}$ and $0-1.8 \mathrm{~dB}$ for CPE heights of $2 \mathrm{~m}$ and $4 \mathrm{~m}$ respectively. At CPE height of $2 \mathrm{~m}$, the accuracy of the prediction increases as the distance increases between the CPE and the transmitter. At CPE height of $4 \mathrm{~m}$, the standard deviation of errors increase as distance from the transmitter is increased, indicating that the path loss prediction accuracy reduces when the CPE is further away from the transmitter.

For both models, the accuracy of the optimised models in predicting the path loss shows superior performance to that of the COST-231 Hata model. The standard deviation of error analysis for the COST-231 Hata model (Table 6) shows that the error range of the COST-231 Hata models is between 29-39dB for suburban environments and 25-34dB for open urban environments. 


\section{Contribution and uniqueness of work}

This paper outlines how an optimised model for the prediction of path loss for WiMAX signal in the $2360-2390 \mathrm{MHz}$ band is developed based on measured field strength in Cyberjaya, Malaysia. After the comparison with measured path loss against theoretical path loss values was done, the best model was developed based on the existing COST-231 Hata model. This model was selected for the optimisation of the measured data because the path loss exponents estimated by the COST-231 Hata model was the closest to the measured path loss exponent. The developed optimised model was validated against the measured field strength, and was found to predict path loss in this band with higher accuracy than the COST-231 Hata model.

The development of these optimised models are crucial because the COST-231 Hata model is developed for the prediction of path loss for up to $2000 \mathrm{MHz}$. Given the emphasis for broadband deployment in Malaysia, the optimised model presented in this paper can be used to predict path loss in the $2360-2390 \mathrm{MHz}$ for WiMAX signals with high accuracy.

\section{Recommendation for future research}

In this study, field data is only available for CPE heights of up till $4 \mathrm{~m}$. Future works can be done by collecting field data for greater CPE heights to verify the accuracy of the proposed optimised model in suburban and open urban environments. The field strength can also be collected in a typical urban environment, and similar methods can be employed to optimise a model based on COST-231 Hata, if found to be applicable, in the $2360-2390 \mathrm{MHz}$ band for the prediction of WiMAX path loss. The proposed method can also be applied to optimise a new model for the prediction of path loss experienced by mobile WiMAX systems.

\section{Conclusion}

Field strength of WiMAX signals in Cyberjaya is collected using a fixed WiMAX receiver and translated into path loss. The WBSs of the BS covers a mix of suburban and open urban environments. Open urban environment is less urban than a typical urban environment. The measured path loss, when compared against theoretical values from the SUI, COST-231 Hata and Egli path loss models, showed the closest agreement with the path loss predicted by the COST-231 Hata model in terms of path loss exponent prediction and standard deviation error analysis. Based on this, an optimised Hata model for the prediction of path loss experienced by WiMAX signals in the $2360-2390 \mathrm{MHz}$ band in suburban and open urban environment is developed. The optimised model showed high accuracy and is able to predict path loss with smaller standard deviation errors as compared to the COST-231 Hata model. It should be noted that the optimised models have a very small operating frequency range, which is between $2360-2390 \mathrm{MHz}$ only. Thus, the models were optimised to be independent of the operating frequency and the height of the BS, as long as the path loss estimation is done within the stipulated operating frequency range.

\section{References}

Abhayawardhana, V. S., Wassell, I. J., Crosby, D., Sellars M.P. \& Brown, M.G. (2005). Comparison of empirical propagation path loss models for fixed wireless access systems. Proceedings of IEEE Conference on Vehicular Technology, Stockholm, Sweden, Vol. 1, pp 73-77

Anderson, H. R (2003). Fixed Broadband Wireless System Design. John Wiley \& Co.

Andrews, J.G., Ghosh, A. \& Muhamed R. (2000). Fundamentals of WiMAX: Understanding Broadband Wireless Networking. Prentice Hall.

Asztalos, T. (2008). Planning a WiMAX Radio Network with A9155. Alcatel-Lucent (April, 2008) COST Action 231 (1999). Digital mobile radio towards future generation systems, final report, tech. rep., European Communities, EUR 18957.

Belloul, B., Aragon-Zaval, A., Saunders, S. R. (2009). Measurements and comparison of WiMAX radio coverage at $2.5 \mathrm{GHz}$ and 3.5GHz. EuCAP 2009, 3rd European Conference on Antennas and Propagation, Berlin.

Egli, J. J. (1957). Radio Propagation above 40 Mc Over Irregular Terrain. Proc. IRE, pp.1383-1391

Erceg, V. \& Greenstein, L. J. (1999). An empirically based path loss model for wireless channels in suburban environments. IEEE Journal on Selected Areas of Communications, vol. 17, pp. 1205-1211

Erceg,V. \& Hari, K. V. S. (2001). Channel models for fixed wireless applications. tech. rep., IEEE 802.16Broadband Wireless Access Working Group.

Hata, M. (1981). Empirical formula for propagation loss in land mobile radio services. IEEE Transactions on Vehicular Technology, vol. VT-29, pp. 317-325. 
Jacques, L. \& Michel, S (2000). Radio Wave Propagation Principles and Techniques. John Wiley \& sons Ltd.

Okumura, Y. (1968). Field strength and it's variability in VHF and UHF land-mobile radio-services. Review of the Electrical Communications Laboratory, vol. 16.

Rao, T. R., Bhaskara Rao S.V., Prasad, M.V.S.N., Sain, M. , Iqbal, A. , \& Lakshmi , D.R. (2000). Mobile Radio Propagation Path Loss Studies at VHF/UHF Bands in Southern India. IEEE Trans. on Broadcasting, Vol.46, No. 2.

Rappaport, T. S. (2002). Wireless Communications, Principles \& Practice. (2nded.). Prentice Hall.

Rial, V., Kraus, H., Hauck, J., \& Buchholz, M. (2007). Measurements and analysis of aWimax field trial at $3.5 \mathrm{GHz}$ in an urban environment. Proceedings of IEEE InternationalSymposium on Broadband

Multimedia Systems and Broadcasting (BMSB '07), Orlando, Fla,USA.

Senza Fili Consulting (for WiMAX Forum) (2005). Fixed, nomadic, portable and mobile applications for 802.16-2004 and 802.16e WiMAX networks. (November, 2005).

Town \& Country Planning Department, Malaysia (2005). Urban Design Guidelines for Cyberjaya, Malaysia.

Wikipedia (2010).Path Loss. [Online] Available: http://en.wikipedia.org/wiki/Path_loss (May 2010)

WiMAX Forum (2009). WiMAX Success Story: How Packet One (P1) Did It. [Online] Available: http://www.p1.com.my/common/pdf/PI_Case_study_WiMAX.PDF (November 2009)

Table 1. Constants for determination of $\gamma$ for SUI model [Erceg, V. \& Greenstein, L.J. ,1999]

\begin{tabular}{|c|c|c|c|c|}
\hline Terrain & Equivalent Environment & $\mathrm{a}$ & $\mathrm{b}$ & $\mathrm{c}$ \\
\hline $\mathrm{A}$ & Urban & 4.6 & 0.0075 & 12.6 \\
\hline $\mathrm{B}$ & Suburban & 4.0 & 0.0065 & 17.1 \\
\hline
\end{tabular}

a, b, c, constants given in Erceg, V. \& Greenstein, L. J. (1999) for calculation of path loss exponents for the Stanford University Interim (SUI) model

Table 2. Field data and calculations of measured and theoretical path loss

\begin{tabular}{|c|c|c|c|c|c|c|c|c|c|c|}
\hline & & & \multicolumn{8}{|c|}{ Calculated path loss using Equations (1), (2), (5) and (11) } \\
\hline & d & RSS & $\mathrm{LS}, 2 \mathrm{~m}$ & $\mathrm{LS}, 4 \mathrm{~m}$ & SUI, $2 \mathrm{~m}$ & SUI, $4 \mathrm{~m}$ & $\begin{array}{c}\mathrm{COST}, \\
2 \mathrm{~m}\end{array}$ & $\begin{array}{c}\mathrm{COST}, \\
4 \mathrm{~m}\end{array}$ & Egli, $2 \mathrm{~m}$ & Egli, $4 \mathrm{~m}$ \\
\hline \multirow{14}{*}{ 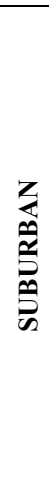 } & 100 & -63.79 & 75.51 & & 121.76 & & 104.75 & & 56.45 & \\
\hline & 150 & -65.25 & 80.16 & & 129.80 & & 111.07 & & 63.50 & \\
\hline & 200 & -67.26 & 83.46 & & 135.51 & & 115.55 & & 68.49 & \\
\hline & 250 & -70.73 & 86.02 & & 139.94 & & 119.03 & & 72.37 & \\
\hline & 300 & -72 & 88.12 & & 143.56 & & 121.88 & & 75.54 & \\
\hline & 350 & -74.5 & 89.89 & & 146.62 & & 124.28 & & 78.22 & \\
\hline & 400 & -75.82 & 91.42 & & 149.27 & & 126.36 & & 80.54 & \\
\hline & 450 & -77.26 & 92.77 & & 151.61 & & 128.20 & & 82.58 & \\
\hline & 500 & -78.39 & 93.98 & & 153.70 & & 129.84 & & 84.41 & \\
\hline & 550 & -79.93 & 95.07 & & 155.60 & & 131.33 & & 86.07 & \\
\hline & 600 & -80.68 & 96.07 & & 157.32 & & 132.69 & & 87.58 & \\
\hline & 650 & -82.1 & 96.99 & & 158.91 & & 133.93 & & 88.97 & \\
\hline & 700 & -83.99 & 97.84 & & 160.38 & & 135.09 & & 90.26 & \\
\hline & 750 & -85.27 & 98.63 & & 161.75 & & 136.17 & & 91.46 & \\
\hline
\end{tabular}




\begin{tabular}{|c|c|c|c|c|c|c|c|c|c|c|}
\hline & 800 & -86.31 & 99.37 & & 163.03 & & 137.17 & & 92.58 & \\
\hline & 850 & -87.68 & 100.07 & & 164.24 & & 138.12 & & 93.63 & \\
\hline & 900 & -89.97 & 100.72 & & 165.37 & & 139.01 & & 94.62 & \\
\hline & 950 & -91.34 & 101.34 & & 166.45 & & 139.85 & & 95.56 & \\
\hline & 1000 & -92.91 & 101.93 & & 167.46 & & 140.65 & & 96.45 & \\
\hline & 100 & -63.87 & & 75.61 & & 118.50 & & 101.81 & & 50.43 \\
\hline & 150 & -64.22 & & 80.00 & & 126.55 & & 108.14 & & 57.48 \\
\hline & 200 & -65.77 & & 83.12 & & 132.26 & & 112.62 & & 62.47 \\
\hline & 250 & -70.73 & & 85.53 & & 136.69 & & 116.10 & & 66.35 \\
\hline & 300 & -71.25 & & 87.51 & & 140.31 & & 118.95 & & 69.52 \\
\hline & 350 & -73.08 & & 89.18 & & 143.37 & & 121.35 & & 72.20 \\
\hline & 400 & -74.52 & & 90.62 & & 146.02 & & 123.43 & & 74.51 \\
\hline & 450 & -76.66 & & 91.90 & & 148.36 & & 125.27 & & 76.56 \\
\hline & 500 & -78.18 & & 93.04 & & 150.45 & & 126.91 & & 78.39 \\
\hline & 550 & -79.99 & & 94.07 & & 152.34 & & 128.40 & & 80.05 \\
\hline & 600 & -80.45 & & 95.01 & & 154.07 & & 129.75 & & 81.56 \\
\hline & 650 & -80.86 & & 95.88 & & 155.66 & & 131.00 & & 82.95 \\
\hline & 700 & -81.06 & & 96.68 & & 157.13 & & 132.16 & & 84.24 \\
\hline & 750 & -82.06 & & 97.43 & & 158.50 & & 133.23 & & 85.43 \\
\hline & 800 & -84.46 & & 98.13 & & 159.78 & & 134.24 & & 86.56 \\
\hline & 850 & -86.39 & & 98.79 & & 160.99 & & 135.19 & & 87.61 \\
\hline & 900 & -87.17 & & 99.40 & & 162.12 & & 136.08 & & 88.60 \\
\hline & 950 & -89.76 & & 99.99 & & 163.19 & & 136.92 & & 89.54 \\
\hline & 1000 & -91.81 & & 100.54 & & 164.21 & & 137.72 & & 90.43 \\
\hline \multirow{38}{*}{ 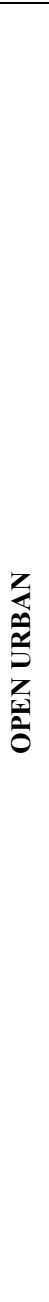 } & 100 & -63.82 & 75.51 & & 121.76 & & 107.23 & & 56.49 & \\
\hline & 150 & -63.99 & 81.42 & & 130.48 & & 113.55 & & 63.53 & \\
\hline & 200 & -64.58 & 85.61 & & 136.68 & & 118.04 & & 68.53 & \\
\hline & 250 & -72.86 & 88.86 & & 141.48 & & 121.52 & & 72.41 & \\
\hline & 300 & -73.83 & 91.52 & & 145.40 & & 124.36 & & 75.58 & \\
\hline & 350 & -73.96 & 93.76 & & 148.72 & & 126.77 & & 78.25 & \\
\hline & 400 & -74.2 & 95.71 & & 151.60 & & 128.85 & & 80.57 & \\
\hline & 450 & -74.69 & 97.42 & & 154.13 & & 130.69 & & 82.62 & \\
\hline & 500 & -82.5 & 98.96 & & 156.40 & & 132.33 & & 84.45 & \\
\hline & 550 & -83.81 & 100.35 & & 158.45 & & 133.81 & & 86.11 & \\
\hline & 600 & -85.33 & 101.62 & & 160.33 & & 135.17 & & 87.62 & \\
\hline & 650 & -87 & 102.78 & & 162.05 & & 136.42 & & 89.01 & \\
\hline & 700 & -88.55 & 103.86 & & 163.64 & & 137.58 & & 90.29 & \\
\hline & 750 & -89.65 & 104.87 & & 165.13 & & 138.65 & & 91.49 & \\
\hline & 800 & -90.26 & 105.81 & & 166.52 & & 139.66 & & 92.61 & \\
\hline & 850 & -93.72 & 106.69 & & 167.82 & & 140.60 & & 93.67 & \\
\hline & 900 & -93.97 & 107.52 & & 169.05 & & 141.49 & & 94.66 & \\
\hline & 950 & -94.5 & 108.31 & & 170.22 & & 142.34 & & 95.60 & \\
\hline & 1000 & -96.09 & 109.06 & & 171.32 & & 143.14 & & 96.49 & \\
\hline & 100 & -63.55 & & 75.61 & & 118.50 & & 101.21 & & 50.47 \\
\hline & 150 & -64.16 & & 81.18 & & 127.23 & & 107.53 & & 57.51 \\
\hline & 200 & -66.03 & & 85.14 & & 133.42 & & 112.02 & & 62.51 \\
\hline & 250 & -67.56 & & 88.20 & & 138.23 & & 115.50 & & 66.39 \\
\hline & 300 & -72.88 & & 90.71 & & 142.15 & & 118.34 & & 69.56 \\
\hline & 350 & -73.01 & & 92.83 & & 145.47 & & 120.74 & & 72.23 \\
\hline & 400 & -75.02 & & 94.66 & & 148.35 & & 122.83 & & 74.55 \\
\hline & 450 & -77.23 & & 96.28 & & 150.88 & & 124.66 & & 76.60 \\
\hline & 500 & -80.11 & & 97.73 & & 153.15 & & 126.31 & & 78.43 \\
\hline & 550 & -81.93 & & 99.04 & & 155.20 & & 127.79 & & 80.08 \\
\hline & 600 & -83.47 & & 100.24 & & 157.07 & & 129.15 & & 81.60 \\
\hline & 650 & -85 & & 101.34 & & 158.80 & & 130.40 & & 82.99 \\
\hline & 700 & -87.9 & & 102.36 & & 160.39 & & 131.55 & & 84.27 \\
\hline & 750 & -86.95 & & 103.30 & & 161.88 & & 132.63 & & 85.47 \\
\hline & 800 & -88.21 & & 104.19 & & 163.27 & & 133.64 & & 86.59 \\
\hline & 850 & -88.35 & & 105.03 & & 164.57 & & 134.58 & & 87.65 \\
\hline & 900 & -89.63 & & 105.81 & & 165.80 & & 135.47 & & 88.64 \\
\hline & 950 & -92.65 & & 106.55 & & 166.97 & & 136.32 & & 89.58 \\
\hline & 1000 & -93.28 & & 107.26 & & 168.07 & & 137.12 & & 90.47 \\
\hline
\end{tabular}

d, distance from transmitter in meters; RSS, Received Signal Strength; LS, Least Square model

(measured path loss); SUI, Standard University Interim model; COST, COST-231 Hata model; Egli,

Egli model; 2m, CPE height 2m; 4m, CPE height 4m 
Table 3. Measured path loss exponents from slope of measured path loss vs. distance graph

\begin{tabular}{|c|c|c|c|c|}
\hline \multirow{2}{*}{} & \multicolumn{2}{|c|}{ SUBURBAN } & \multicolumn{2}{c|}{ OPEN URBAN } \\
\cline { 2 - 5 } & $2 \mathrm{~m}$ & $4 \mathrm{~m}$ & $2 \mathrm{~m}$ & $4 \mathrm{~m}$ \\
\hline Path loss exponent & 2.642 & 2.493 & 3.355 & 3.165 \\
\hline
\end{tabular}

2m, CPE height 2m; 4m, CPE height $4 \mathrm{~m}$

Table 4. Measured and theoretical path loss exponents

\begin{tabular}{|c|c|c|c|c|}
\hline \multirow{2}{*}{ Path Loss Model } & \multicolumn{2}{|c|}{ SUBURBAN, $\mathrm{n}$} & \multicolumn{2}{c|}{ OPEN URBAN, $\mathrm{n}$} \\
\cline { 2 - 5 } & $2 \mathrm{~m}$ & $4 \mathrm{~m}$ & $2 \mathrm{~m}$ & $4 \mathrm{~m}$ \\
\hline LS (measured path loss) & 2.642 & 2.493 & 3.355 & 3.165 \\
\hline SUI & 4.571 & 4.571 & 4.957 & 4.957 \\
\hline COST-231 & 3.591 & 3.591 & 3.591 & 3.591 \\
\hline Egli & 4.000 & 4.000 & 4.000 & 4.000 \\
\hline
\end{tabular}

LS, Least Square (measured path loss); SUI , Stanford University Interim model; Egli, Egli model; COST-231 Hata, COST-231 Hata model; 2m, CPE height 2m; 4m, CPE height 4m; n, path loss exponent

Table 5. Comparison of measured and COST-231 Hata path loss exponents with optimised model

\begin{tabular}{|c|c|c|c|c|}
\hline \multirow{2}{*}{ Path Loss Model } & \multicolumn{2}{|c|}{ SUBURBAN } & \multicolumn{2}{c|}{ OPEN URBAN } \\
\cline { 2 - 5 } & $2 \mathrm{~m}$ & $4 \mathrm{~m}$ & $2 \mathrm{~m}$ & $4 \mathrm{~m}$ \\
\hline LS (measured path loss) & 2.642 & 2.493 & 3.355 & 3.165 \\
\hline COST-231Hata model & 3.591 & 3.591 & 3.591 & 3.591 \\
\hline Optimised model & 2.180 & 2.180 & 3.346 & 3.346 \\
\hline
\end{tabular}

2m, CPE height 2m; 4m, CPE height $4 \mathrm{~m}$ 
Table 6. Standard deviation of errors between measured path loss with COST-231 Hata path loss and measured path loss with optimised model predicted path loss

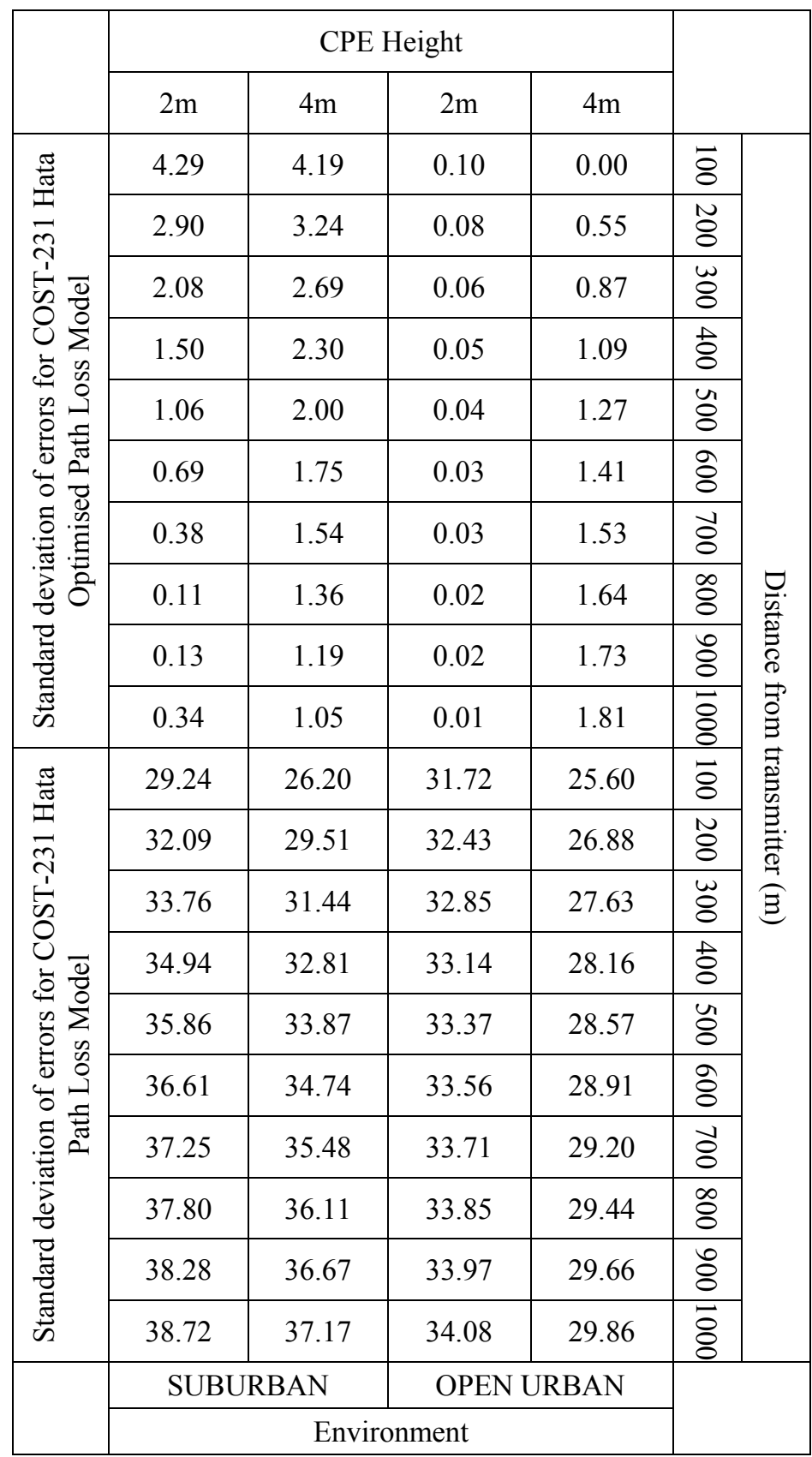

2m, CPE height 2m; 4m, CPE height 4m; CPE, Customer Premise Equipment 


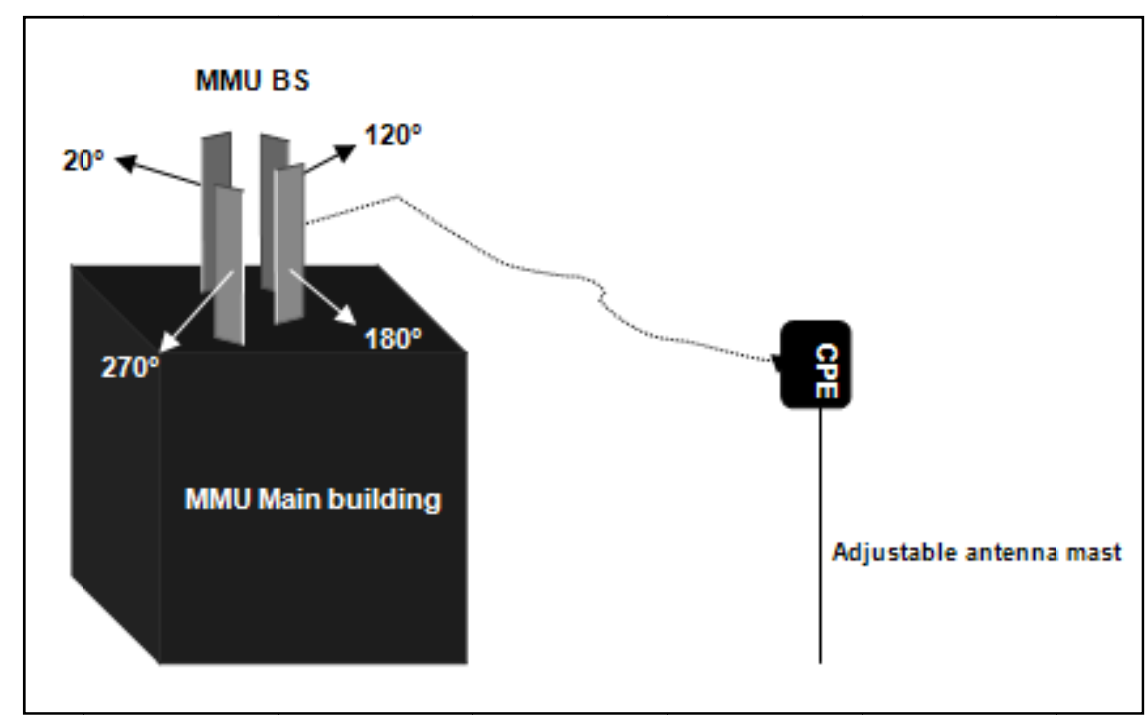

Figure 1. Experimental setup for field testing

MMU BS, MMU Base Station; CPE, Customer Premise Equipment; $\mathbf{2 0}^{\circ}, \mathbf{1 2 0}^{\circ}, \mathbf{1 8 0}^{\circ}, \mathbf{2 7 0}^{\circ}$, WiMAX Base Station azimuths

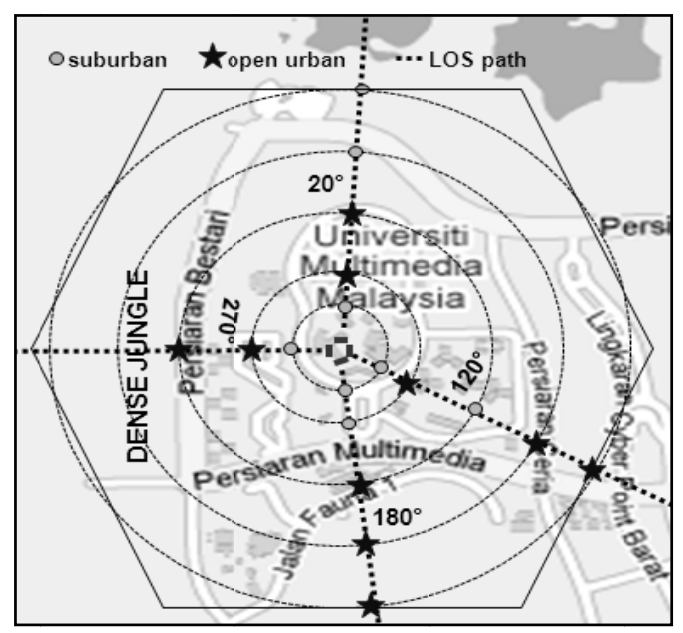

Figure 2. The estimated coverage area of the MMU BS and the LOS path of each WBS.

LOS, Line of Sight 

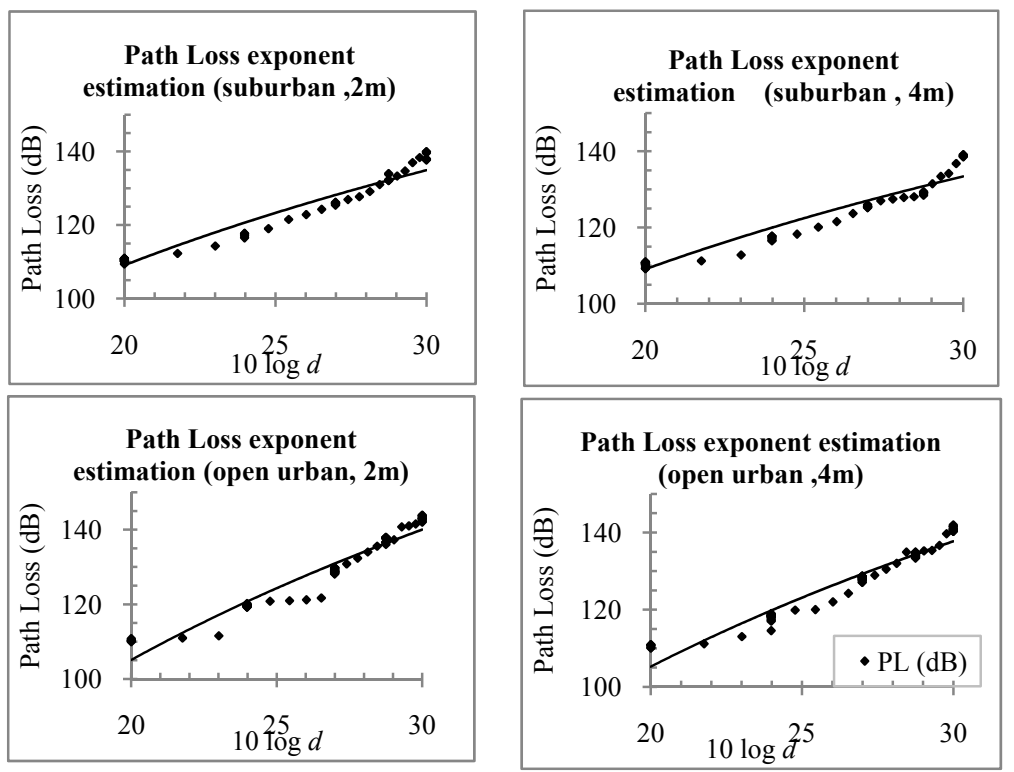

Figure 3. Path Loss $(\mathrm{dB})$ versus distance $(\mathrm{m})$ for open and suburban environments with CPE heights $2 \mathrm{~m}$ and $4 \mathrm{~m}$

PL, path loss; d, distance from transmitter (m)

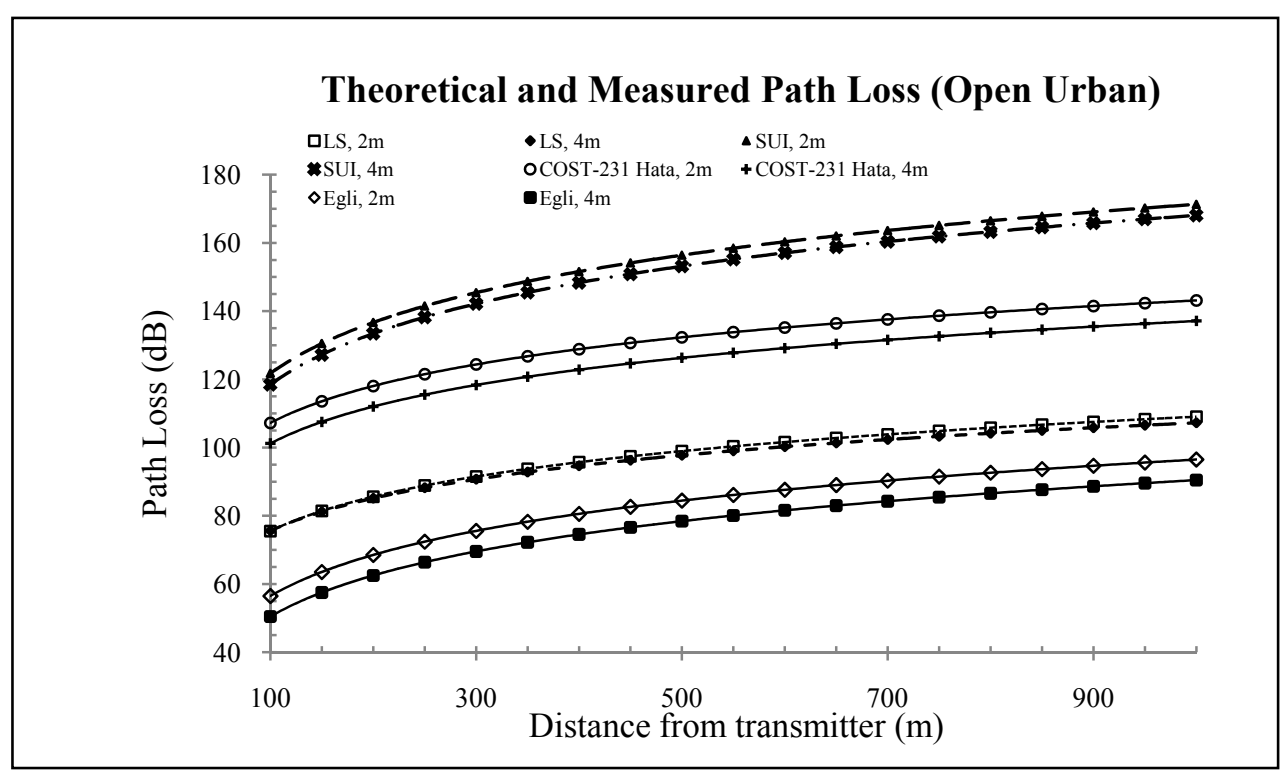

Figure 4. Comparison of measured and theoretical path loss in open urban environments for CPE heights of $2 \mathrm{~m}$ and $4 \mathrm{~m}$

LS, Least Square (measured path loss); SUI , Stanford University Interim model; Egli, Egli model; COST-231

Hata, COST-231 Hata model; 2m, CPE height 2m; 4m, CPE height $4 m$ 
Theoretical and Measured Path Loss (Suburban)

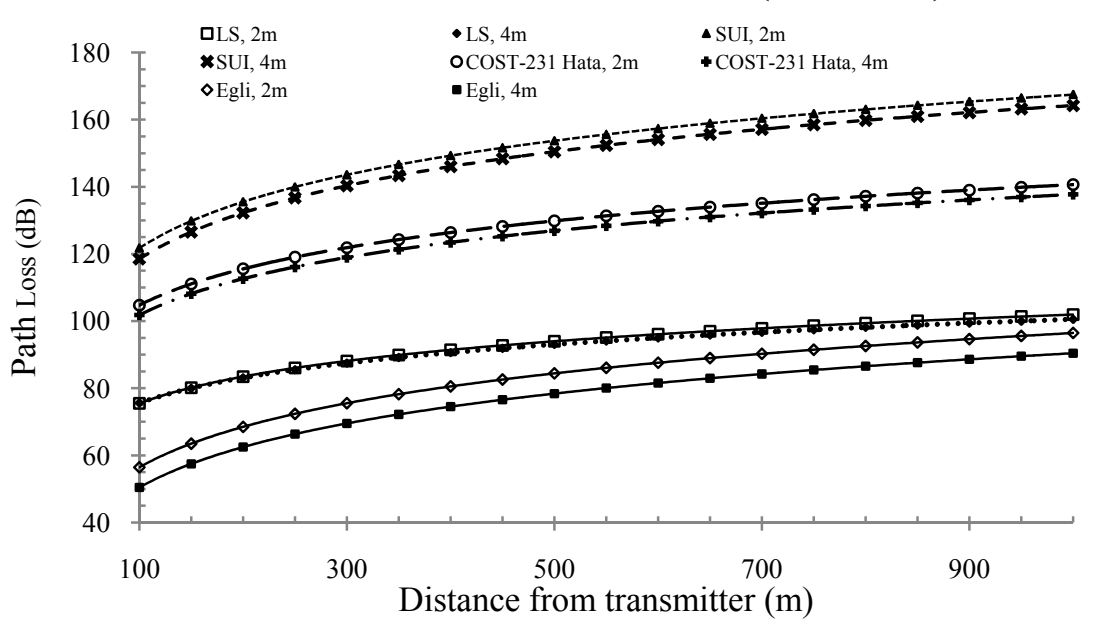

Figure 5. Comparison of measured and theoretical path loss in suburban environments

LS, Least Square (measured path loss); SUI , Stanford University Interim model; Egli, Egli model; COST-231

Hata, COST-231 Hata model; 2m, CPE height 2m; 4m, CPE height $4 \mathrm{~m}$

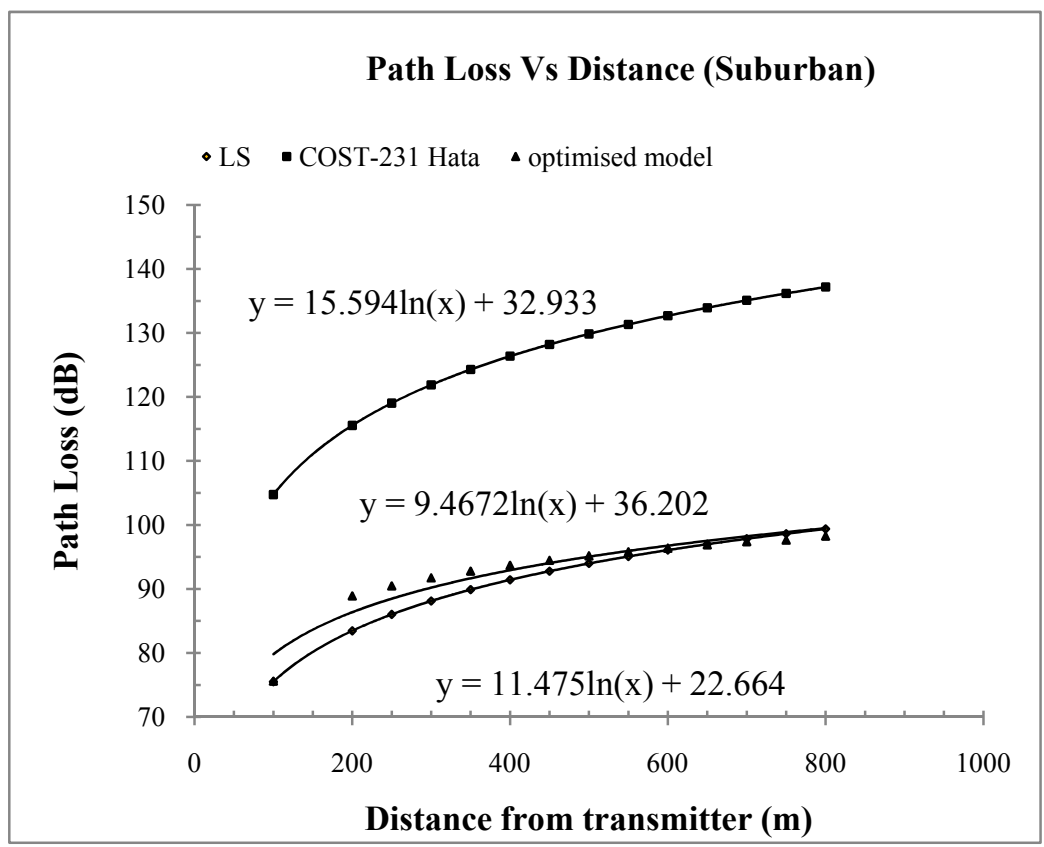

Figure 6. Development of optimised model based on COST-231 Hata model for suburban environment LS, Least Square model (measured path loss); COST-231 Hata, COST-231 Hata model 


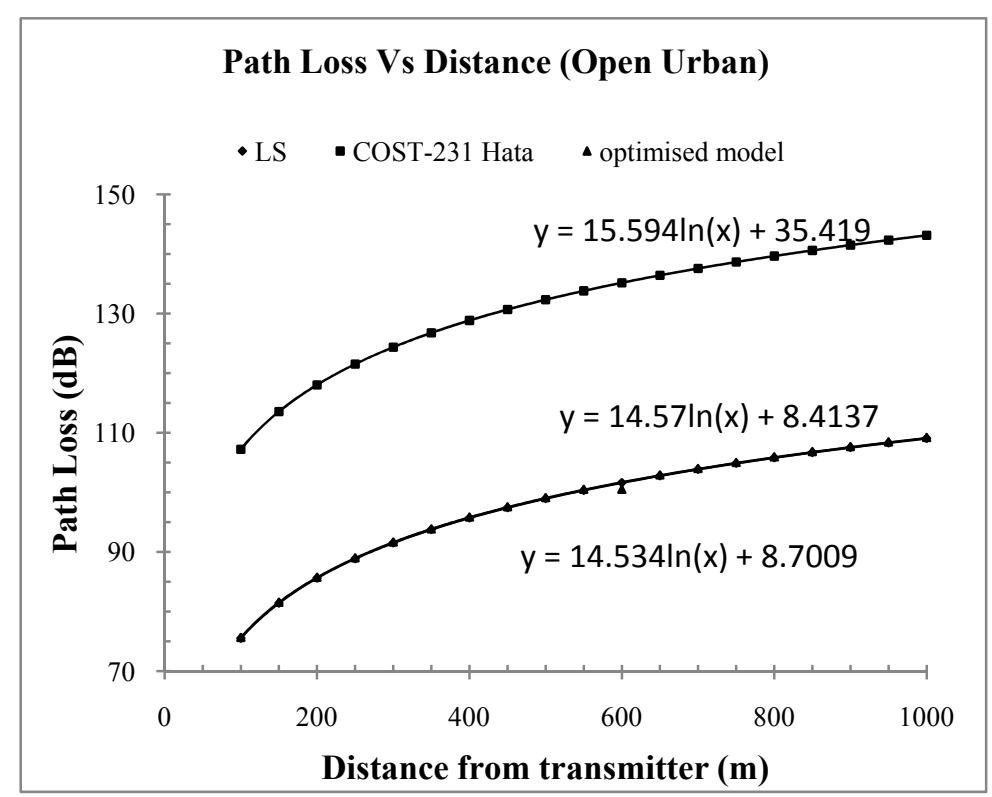

Figure 7. Development of optimised model based on COST-231 Hata model for open urban environment LS, Least Square model (measured path loss); COST-231 Hata, COST-231 Hata model

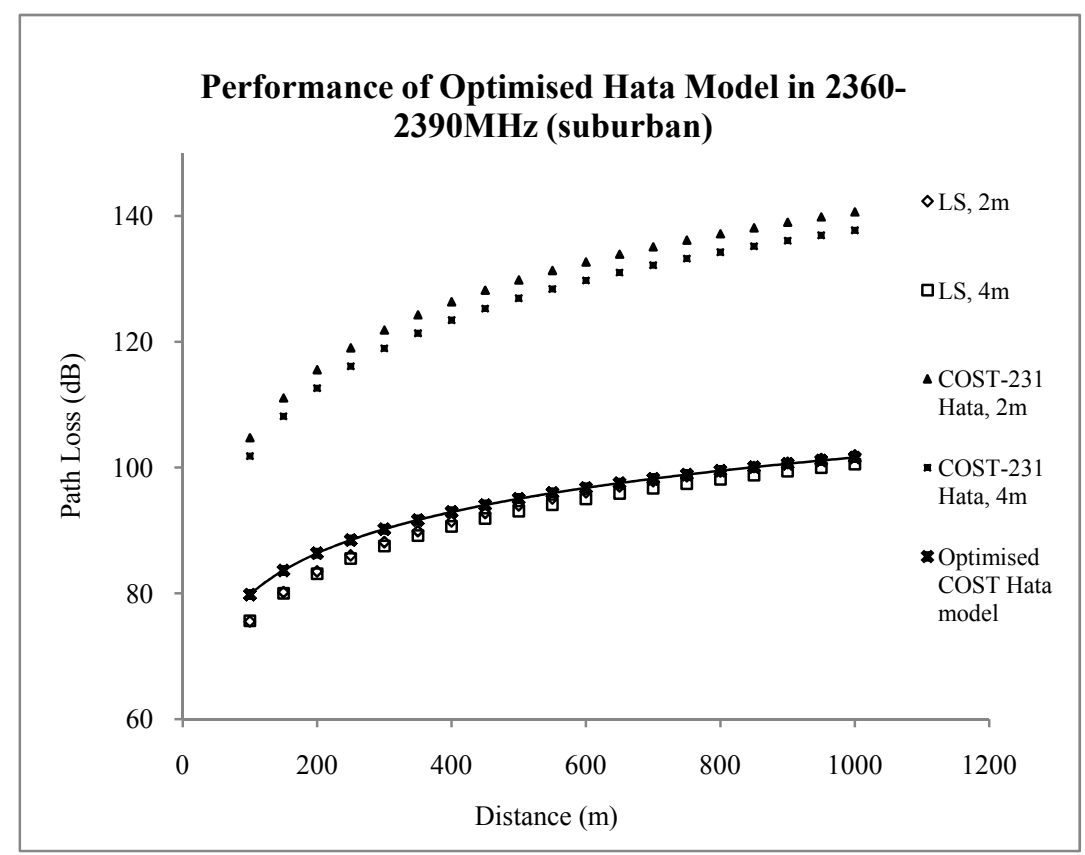

Figure 8. Comparison of path loss prediction by optimised model in suburban environment

LS, Least Square model (measured path loss); 2m, CPE height 2m; 4m, CPE height 4m 


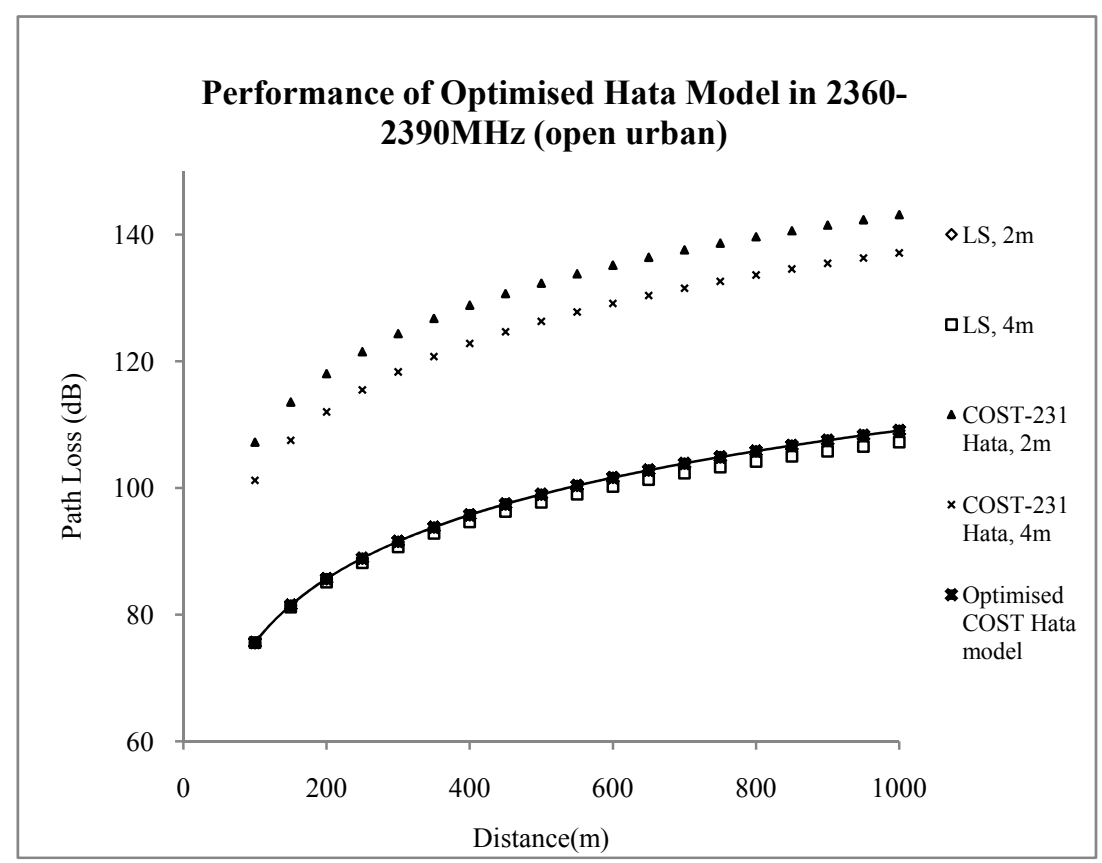

Figure 9. Comparison of path loss prediction by optimised model in suburban environment LS, Least Square model (measured path loss)

LS, Least Square model (measured path loss); 2m, CPE height 2m; 4m, CPE height 4m 